\title{
Can Neurons Sense Millimeter Waves?
}

\author{
Peter H. Siegel ${ }^{\mathrm{a}}$ and Victor Pikov ${ }^{\mathrm{b}}$ \\ ${ }^{a}$ California Institute of Technology, Pasadena, CA 91125 USA \\ ${ }^{\mathrm{b}}$ Huntington Medical Research Institute, Pasadena, CA 91105 USA
}

\begin{abstract}
The applications and commercial use of millimeterand submillimeter-wavelength radiation are becoming more and more widespread in our society. As such, there is a growing need to better understand and quantify both the coupling mechanisms and the impact of this long wavelength energy on biological function. Independent of the health impact of high doses of radio frequency (RF) energy on full organisms, there exists the potential for more subtle effects, which can best be quantified in studies which examine real-time changes in cellular function as RF energy is applied. In this paper we present the first real time examination of millimeter-wave induced changes in cellular activity at absorbed power levels well below the existing safe exposure limits of $1 \mathrm{~mW} / \mathrm{cm}^{2}$. Using sensitive patch clamp measurements on pyramidal neurons in cortical slices of neonatal rats, the authors present the first direct evidence of millimeter-wave induced changes in action potential firing rates and membrane porosity. Non-invasive modulation of neuronal activity could prove useful in a variety of health applications from suppression of peripheral neuropathic pain to treatment of central neurological disorders.
\end{abstract}

\section{BACKGROUND}

I $\mathrm{N}$ a prior study [1] the authors presented a series of experiments on an in vitro immortalized epithelial cell line (designated H1299) that correlated changes in membrane permeability with exposure to modest levels of millimeter-wave power: $\sim 10 \mathrm{~mW} / \mathrm{cm}^{2}$ for two minutes at 50 $\mathrm{GHz}$. These measurements involved the transfection of a pEGFP-F (farnesylated green fluorescent protein) plasmid onto the inner leaflet of the cell membrane's lipid bi-layer. Upon exposure to blue light $(490 \mathrm{~nm})$ the fGFP fluoresces green (520nm), lighting up the inner leaflet (Fig. 1-top). When oxonol is added to the media it binds to the outer membrane. The opening of pores in the membrane allows the outer bound oxonol to reach into the inner leaflet and serve as a quenching agent for the GFP through a FRET (Förster resonance energy transfer) process. The resultant fluorescent signature changes from green to red $(620 \mathrm{~nm})$ indicating the appearance of membrane pores (Fig. 1-bottom). After the millimeter-wave power was turned off the fluorescent signature reverted back to pre-exposure levels, showing transient behavior (Fig. 2). The bath temperature was monitored during the exposure and did not rise more than $3{ }^{\circ} \mathrm{C}$. This first set of experiments gave us an indication that millimeter-waves can have a significant effect on cell function without a large rise in ambient temperature.

\section{EXPERIMENTS ON NEURONS}

The study begun in [1] was extended to a neuronal cell line (B104 - a gift from Peter W. Vanderklish, Scripps Institute), again transfected with a pEGFP-F plasmid expressing fGFP. A similar FRET experiment was performed using $10 \mathrm{~mW} / \mathrm{cm}^{2}$ at $60 \mathrm{GHz}$ for two minutes, and the resultant shift in fluorescent signal was recorded with a pair of ocular mounted photomultipliers each with a narrowband filter centered on 520 and $620 \mathrm{~nm}$. The same increase in the red signature (indicating the appearance of membrane pores) was recorded but no return to pre-exposure state was seen. Subsequent discussions with the inventors of the pEGFP-F/oxonol FRET technique (Professors Vergara and DiFranco, UCLA) pointed towards possible enhanced solubility of oxonol in PBS upon millimeter-wave exposure resulting in a permanent increase in the red background signal.
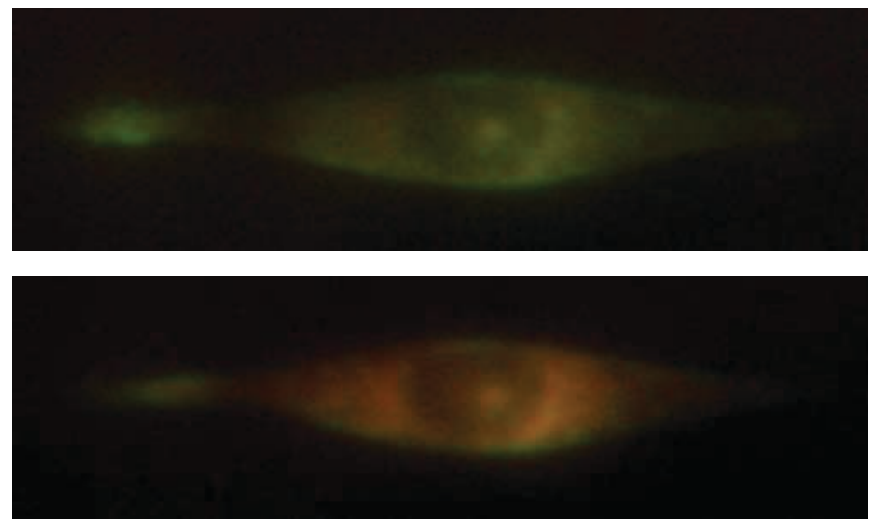

Fig 1. Top. Photograph of single H1299 cell in oxonol and phosphate buffered saline expressing lipid bound fGFP at cell membrane (transfected with pEGFP-F vector) before millimeter wave exposure. Bottom: Same cell after exposure. Red color indicates FRET-induced oxonol fluorescence.

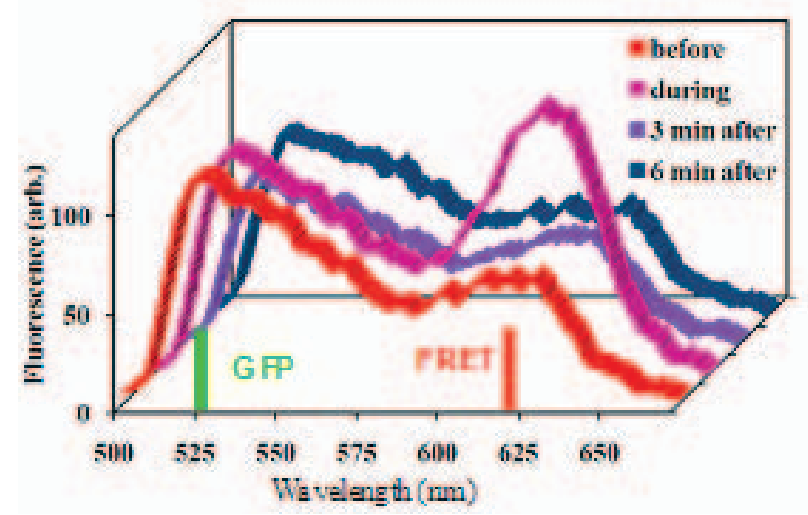

Fig. 2. H1299 cell spectrum (green GFP \& red FRET lines) before during and after exposure to $\sim 1-3 \mathrm{~mW} / \mathrm{cm}^{2} \mathrm{RF}$ energy at $50 \mathrm{GHz}$ for two minutes. $620 \mathrm{~nm}$ red FRET line indicates membrane depolarization (open). $520 \mathrm{~nm}$ Green GFP line indicates normal membrane signature. Note membrane depolarizes then repolarizes after 3 minutes. 


\section{PATCh Clamp EXPERIMENTS ON CORTICAL SliceS}

In order to be able to record real time changes on ex vivo neurons, a series of patch-clamp experiments using 13-16d rat pup cortical slices was arranged with the help of Dr. Mike Harrington and Dr. Zianghing Arakaki at the Huntington Medical Research Institutes. The experimental arrangement is shown in Fig. 3. 300 micron thick vibrotome slices containing
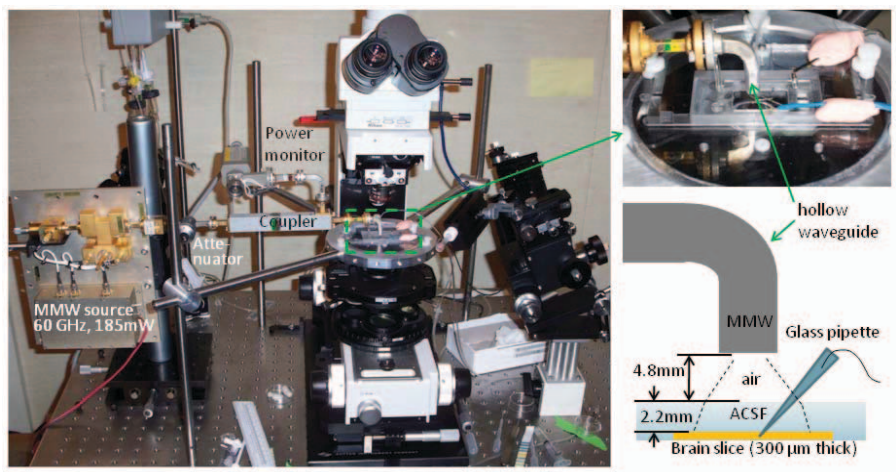

Fig. 3. Left. Photograph of patch clamp measurement set up with vertically coupled RF power source. Above right. Close up showing waveguide and tissue chamber. Below right. Schematic showing probe geometry and distances used for calculating the beam profile and power distribution at the tissue slice.

the cerebral cortex were placed in the tissue chamber and incubated at room temperature in artificial cerebrospinal fluid (ACSF) (approximately $3 \mathrm{ml}$ ) with bubbled oxygen (95\%) and $\mathrm{CO}_{2}(5 \%)$ at a $\mathrm{pH}$ of 7.4 . The electrical probe pipette was filled mostly with K-glutonate, $\mathrm{KCl}$ and $\mathrm{NaCl}$ (140:5:4) plus a buffer agent (HEPES) to obtain a resistance between 4 and $7 \mathrm{M} \Omega$. The ionically balanced probe was inserted through the membrane and into the head of a single pyramidal neuron and positive current (75pA typical) was applied for five seconds out of every twenty seconds, repeating cyclically for several minutes during each measurement sequence. The intracellular-to-extracellular voltage was recorded continuously and the appearance, firing rate, peak voltage, rise and fall times and input resistance $(\Delta \mathrm{V} / \Delta \mathrm{I})$ of the action potential spikes upon the positive current cycles provided the baseline and RF induced changes that are the basis for the experiments. Millimeter-wave power $(60 \mathrm{GHz}$, with $7.5,15,30,60,120$ and $185 \mathrm{~mW}$ present at the waveguide output port) was introduced in random sequence via a mechanical rotary vane attenuator (no electronic switches were thrown during the measurements), held fixed for one minute (3 current cycles), then turned off. Bath temperature was constantly monitored by an in-situ thermocouple. The temperature rise with power level varied from $<0.1^{\circ} \mathrm{C}$ to a maximum of $3^{\circ} \mathrm{C}$ at the highest setting. Fig. 4 shows sample recordings taken at four different RF power levels from approximately 0.1 to $1 \mu \mathrm{W} / \mathrm{cm}^{2}$ as calculated at the plane of the cortical slice after absorption through $2.2 \mathrm{~mm}$ of ACSF. Changes in the firing rate were observable at power levels of $\sim 0.3 \mu \mathrm{W} / \mathrm{cm}^{2}$ and above. Rise and decay slopes of individual action potentials and membrane resistance (not shown) were also strongly correlated with RF power level indicating opening of the membrane ion channels. These experiments are described in greater detail in [2].

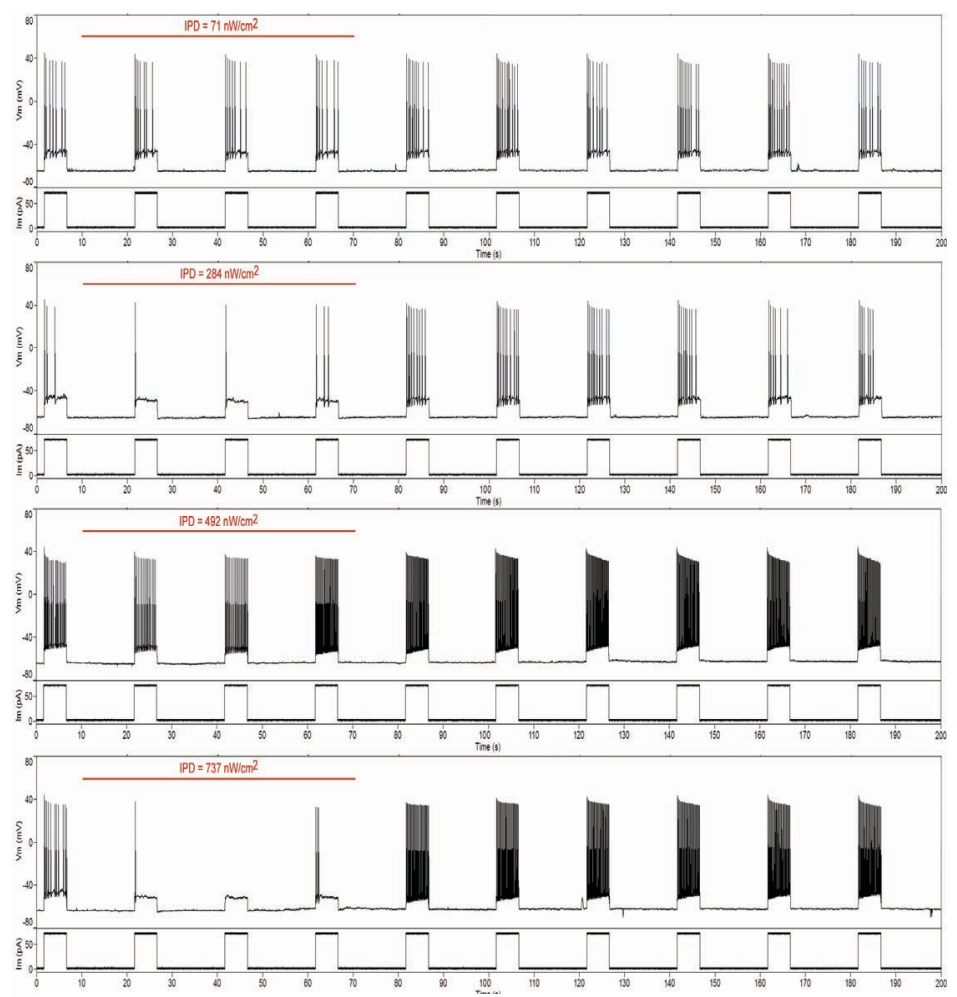

Fig. 4. Sample recording of neuronal activity (membrane voltage, $\mathrm{V}_{\mathrm{m}}$ ) and injected membrane current $\left(\mathrm{I}_{\mathrm{m}}\right)$ versus time at four RF power levels applied for 1 minute. Incident Power Density (IPD) was calculated according to beam spread and loss in the $2.2 \mathrm{~mm}$ of ACSF fluid above the cortical slice. Top to Bottom: 71, 284, 492, 737nW/cm². Red bars indicate the time and duration of exposure. For each sequence above the neuron was stimulated for $5 \mathrm{sec}$ and allowed to rest for $20 \mathrm{sec}$. As the RF power is increased strong inhibition of the neuron action potential firing rate is seen during the exposure. Excitation was also observed. In all cases the neuron returned to pre-exposure firing rates within 6 minutes.

\section{DISCUSSION}

Fig. 4 clearly shows strong correlative behavior between extremely low millimeter-wave power densities (3000 times lower than the U.S. maximum permissible exposure of $1 \mathrm{~mW} / \mathrm{cm}^{2}$ for 6 minutes) and neuronal activity. At power levels of approximately $300 \mathrm{nW} / \mathrm{cm}^{2}$ and above, strong inhibition of the action potential firing rate was observed. Examination of the individual rise and fall time and membrane input resistance also showed strong correlation with increasing power indicating transient changes in membrane porosity. The rise in bath temperature during exposure was $<3^{\circ} \mathrm{C}$ and radiant heating does not produce an equivalent change in the observed parameters [2]. These results are believed to be the first positive correlative measurements of fast (msec) changes in neuronal activity with ultra-low power millimeter wave exposures. Many more experiments are required to cover the available RF stimulus parameter space and to isolate specific neuronal responses as a prelude to clinically significant applications.

\section{REFERENCES}

[1]. P.H. Siegel and V. Pikov, "Impact of Low Intensity Millimeter-Waves on Cell Membrane Permeability," $34^{\text {th }}$ Int. Conf. on Infrared, Millimeter, and Terahertz Waves, Busan, Korea, Sept. 2009.

[2]. V. Pikov, X. Arakaki, M. Harrington, S.E. Fraser and P.H. Siegel, "Modulation of neuronal activity and plasma membrane properties with low-power millimeter waves in organotypic cortical slices," to appear in Journal of Neural Engineering, August 2010 